\title{
Lenalidomide for anemia correction in lower-risk del(5q) myelodysplastic syndrome patients of Asian ethnicity
}

\author{
Junshik Hong ${ }^{1}$, Yoo Jin Lee ${ }^{2}$, Sung Hwa Bae ${ }^{3}$, Jun Ho $\mathrm{Yi}^{4}$, Sungwoo Park ${ }^{5}$, Myung Hee Chang ${ }^{6}$, \\ Young Hoon Park ${ }^{7}$, Shin Young Hyun ${ }^{8}$, Joo-Seop Chung ${ }^{9}$, Ji Eun Jang ${ }^{10}$, Joo Young Jung ${ }^{11}$,
} So-Yeon Jeon ${ }^{12}$, Seo-Young Song ${ }^{13}$, Hawk Kim ${ }^{14}$, Dae Sik Kim ${ }^{15}$, Sung-Hyun Kim ${ }^{16}$, Min Kyoung Kim ${ }^{17}$, Sang Hoon $\mathrm{Han}^{18}$, Seonyang Park ${ }^{19}$, Yoo-Jin Kim ${ }^{20}$, Je-Hwan Lee ${ }^{21}$, on behalf of the AML/MDS Working Party of the Korean Society of Hematology

${ }^{1}$ Department of Internal Medicine, Seoul National University College of Medicine, Seoul National University Hospital, Seoul, ${ }^{2}$ Department of Hematology and Oncology, Ulsan University Hospital, University of Ulsan College of Medicine, Ulsan, ${ }^{3}$ Department of Internal Medicine, Daegu Catholic University School of Medicine, Daegu Catholic University Hospital, Daegu, ${ }^{4}$ Division of Hematology-Oncology, Chung-Ang University Hospital, Seoul, Department of Internal Medicine, ${ }^{5}$ Gyeongsang National University Hospital, Gyeongsang National University School of Medicine, Jinju, ${ }^{6}$ National Health Insurance Service Ilsan Hospital, Goyang, ${ }^{7}$ Ewha Womans University Mokdong Hospital, Ewha Womans University College of Medicine, Seoul, ${ }^{8}$ Yonsei University Wonju College of Medicine, Wonju, ${ }^{9}$ Pusan National University College of Medicine, Pusan National University Hospital, Busan, ${ }^{10}$ Division of Hematology, Department of Internal Medicine, Yonsei University College of Medicine, Seoul, ${ }^{11}$ Department of Internal Medicine, Dongtan Sacred Heart Hospital, Hallym University College of Medicine, Hwaseong, ${ }^{12}$ Division of Oncology and Hematology, Department of Internal Medicine, Jeonbuk National University Hospital-Jeonbuk National University Medical School, Jeonju, ${ }^{13}$ Department of Internal Medicine, Kangwon National University College of Medicine, Kangwon National University Hospital, Chuncheon, ${ }^{14}$ Division of Hematology, Gachon University Gil Medical Center, Gachon University College of Medicine, Incheon, Division of Hematology-Oncology, Department of Internal Medicine, ${ }^{15}$ Korea University Guro Hospital, Seoul, ${ }^{16}$ Dong-A University College of Medicine, Dong-A University Hospital, Busan, ${ }^{17}$ Yeungnam University College of Medicine, Daegu, Department of Internal Medicine, ${ }^{18}$ Jeju National University Hospital, Jeju National University School of Medicine, Jeju, ${ }^{19}$ Inje University Haeundae Paik Hospital, Busan, ${ }^{20}$ Seoul St. Mary's Hematology Hospital, College of Medicine, The Catholic University of Korea, ${ }^{21}$ University of Ulsan College of Medicine, Asan Medical Center, Seoul, Korea

p-ISSN 2287-979X / e-ISSN 2288-0011 https://doi.org/10.5045/br.2021.2021086 Blood Res 2021;56:102-108.

Received on April 23, 2021 Accepted on June 2, 2021

\section{Correspondence to}

\section{Je-Hwan Lee, M.D., Ph.D.}

University of Ulsan College of Medicine, Asan Medical Center, 88, Olympic-ro 43-gil, Songpa-gu, Seoul 05505, Korea E-mail: jhlee3@amc.seoul.kr

(C) 2021 Korean Society of Hematology

\section{Background}

To estimate real-world outcomes in East Asian populations, we conducted a nationwide retrospective analysis of the efficacy and safety of lenalidomide for del $(5 q)$ myelodysplastic syndrome (MDS) patients with transfusion-dependent anemia in Korea.

Methods

Patients aged $\geq 19$ years who had received lenalidomide for the treatment of lower-risk, red blood cell (RBC) transfusion-dependent del(5q) MDS were selected. A filled case report form (CRF) with information from electronic medical records was requested from members of the acute myeloid leukemia (AML)/MDS Working Party of the Korean Society of Hematology. All the CRFs were gathered and analyzed.

Results

A total of 31 patients were included in this study. Of 28 evaluable patients, 19 (67.9\%) achieved RBC transfusion independence (RBC-TI). Female sex and the development of thrombocytopenia during treatment were associated with achieving RBC-TI. The most common non-hematologic toxicities were pruritus, fatigue, and rashes. All non-hematologic toxicities of grades $\geq 3$ were limited to rash $(12.9 \%)$ and pruritus $(6.5 \%)$. Dose reduction was required in 15 of the 19 responders $(78.9 \%)$. The most common final stable dosing schedule for the responders was $5 \mathrm{mg}$ once every other day $(31.6 \%)$.

\section{Conclusion}

Lenalidomide efficacy and tolerability were similar in the Asian del(5q) MDS patients and western patients. Dose reduction during treatment was common, but it was not associated with inferior outcomes.

Key Words $5 q$ deletion syndrome, Myelodysplastic syndrome, Lenalidomide, Anemia

(2) This is an Open Access article distributed under the terms of the Creative Commons Attribution Non-Commercial License (http://creativecommons.org/licenses/by-nc/4.0) 6 which permits unrestricted non-commercial use, distribution, and reproduction in any medium, provided the original work is properly cited. 


\section{INTRODUCTION}

Myelodysplastic syndrome (MDS) is a clonal hematologic stem cell disorder that presents with ineffective hematopoiesis and cytopenia of peripheral blood cells [1]. It is classified into lower- or higher-risk disease according to the degree of cytopenia, amount of bone marrow blasts, and cytogenetic abnormalities [2, 3]. Allogeneic hematopoietic stem cell transplantation is the only curative option for higher-risk MDS that, otherwise, has a dismal prognosis $[4,5]$. For lower-risk MDS, the correction of cytopenia and delay of disease progression to secondary acute myeloid leukemia (AML) are the goals of treatment [1].

The deletion of the long arm of chromosome 5 [del(5q)] is the most common cytogenetic abnormality in patients with MDS at diagnosis [6]. Approximately 10\% of MDS patients harboring del $(5 \mathrm{q})$ have the deletion as the sole cytogenetic abnormality [7], which is classified as 'MDS associated with isolated del(5q)' according to the 2018 World Health Organization (WHO) classification criteria [8]. Some of these patients, mostly with a deletion in the $5 \mathrm{q} 315 \mathrm{q} 33$ chromosomal region, have distinctive features including transfusion-dependent macrocytic anemia, low to normal white blood cell count/absolute neutrophil count (ANC) ratio, and normal to increased platelet count; these characterize the del(5q) syndrome [9].

Lenalidomide is an analog of thalidomide, an immunomodulatory drug. It has been used as a standard treatment for patients with lower-risk MDS harboring del(5q) and red blood cell (RBC) transfusion dependency $[1,7]$. Its effectiveness may be attributed to its unique mechanism of action, which suppresses clonal hematopoietic cells via synthetic lethality mediated by the cereblon-dependent degradation of haplodeficient proteins encoded within the deleted $5 q$ region [10]. This karyotype-specific therapeutic approach can lead to a remarkable improvement in MDS treatment efficacy by achieving a high rate of erythroid response and RBC transfusion independence (RBC-TI) [11, 12] with a possible survival benefit [13].

Although lenalidomide has been approved for del(5q) MDS by the U.S. FDA and the European Medicines Agency in 2005 and 2013, respectively, data on East Asian del(5q) MDS patients are scarce. In East Asian populations, del(5q) MDS is rare, and its reported incidence is low $[7,14]$. In the analysis from the International Working Group for Prognosis of MDS comparing 300 Japanese and 5838 Caucasian MDS patients, the prevalence of del( $(5 q)$ was significantly lower in the Japanese population (1.9\%) than in the Caucasians (8.6\%) [15]. To the best of our knowledge, only one published study has reported the outcomes of 11 Japanese lower-risk MDS patients with del(5q) MDS and symptomatic anemia [16].

In Korea, lenalidomide for del(5q) MDS was approved by the Korea Ministry of Food and Drug Safety (MFDS) in June 2018 and reimbursed by the National Health Insurance Review and Assessment Service (HIRA) in May
2019. To provide real-world evidence on lenalidomide for East Asian MDS patients, particularly focusing on the appropriateness of the recommended dose and dosing schedule of lenalidomide derived from previous studies involving western patients, we conducted a nationwide retrospective analysis of the efficacy and safety of lenalidomide treatment for MDS patients harboring del $(5 q)$ in Korea.

\section{MATERIALS AND METHODS}

\section{Patients and ethics statement}

Patients were included if they were 19 years or older and had ever received lenalidomide (Revlimid) for the treatment of lower-risk RBC transfusion-dependent del(5q) MDS between June 2018 and May 2020. Lower-risk MDS was defined as low- or intermediate-risk according to the International Prognosis Scoring System (IPSS), and RBC transfusion dependency as having received any RBC transfusion within the last 8 weeks and more than 8 units of RBC transfusion within the last 6 months. Patients who had both $\operatorname{del}(5 q)$ and $-7 / \operatorname{del}(7 q)$, those who used lenalidomide for other reasons such as multiple myeloma treatment, and those who had an ANC of $<500 / \mu \mathrm{L}$ or a platelet count of $<25,000 / \mu \mathrm{L}$ were excluded from the analysis.

This study was approved by the Institutional Review Board (IRB) of each participating institution (approval number of the principal investigator's institution, University of Ulsan Asan Medical Center, Seoul, Republic of Korea: 2020-0930). The requirement for informed consent was waived by each IRB, considering the retrospective nature of the current study.

\section{Treatment with lenalidomide}

The recommended starting dose of lenalidomide was 10 mg once daily per os for 21 consecutive days, with rest for 7 days ( 1 cycle $=28$ days). Patients with other starting doses or schedules were included. Dose reduction or interruption was decided according to the discretion of the physicians based on recommendations for responding to hematologic and non-hematologic toxicities $[11,12]$. If the RBC transfusion burden failed to improve with a reduction of $\geq 50 \%$ despite the lenalidomide administration over 4 months, it had to be discontinued according to the HIRA policy.

\section{Data acquisition}

The AML/MDS Working Party of the Korean Society of Hematology actively contacted the members and requested eligible patients. The responding hematologists were provided a protocol and auxiliary documents for the approval of the current study from the IRB of each institution. After approval, a filled case report form was requested, which consisted of items about the demographics of the patients (age, sex, height, and body weight), disease-related information (date of MDS diagnosis, WHO classification, IPSS risk, transfusion history, history of prior treatment, and base- 
line laboratory data including G-banding karyotype), and treatment-related information (date of lenalidomide initiation, blood cell counts at $4,8,16,24$, and 48 th weeks from treatment initiation, response to treatment, hematologic and non-hematologic toxicities according to the Common Terminology Criteria for Adverse Events v5.0, dose reduction or interruption during treatment, treatment discontinuation with the reason for discontinuation, and survival or last follow-up data). Considering the retrospective nature of this study, a window of \pm 4 days was allowed for the reports for the 4 th and 8th weeks. Likewise, windows of \pm 7 days and \pm 14 days were allowed for the reports for the 12 th to 16 th week and thereafter, respectively. The efficacy of lenalidomide was estimated based on the rate of achieving RBC-TI, defined as maintaining RBC transfusion independence for $\geq 8$ weeks during treatment with hematologic improvement-erythroid major (HI-E major) response according to the 2000 International Working Group criteria.

\section{Statistical analysis}

As the current study evaluated the efficacy and safety of a single agent (lenalidomide), most of the statistical analyses were descriptive. The frequency and ratio of the catego-

\begin{tabular}{|c|c|}
\hline Parameter & $\mathrm{N}(\%)$ \\
\hline \multicolumn{2}{|l|}{ Age at lenalidomide initiation $(\mathrm{yr})$} \\
\hline Median (range) & $66(46-83)$ \\
\hline \multicolumn{2}{|l|}{ Sex } \\
\hline Male & $8(25.8)$ \\
\hline Female & $23(74.2)$ \\
\hline \multicolumn{2}{|c|}{$\begin{array}{l}\text { Time from initial MDS diagnosis to lenalidomide } \\
\text { initiation ( } \mathrm{wk} \text { ) }\end{array}$} \\
\hline Median & 56 \\
\hline Range & $0-424$ \\
\hline \multicolumn{2}{|l|}{ Transfusion dependence } \\
\hline At MDS diagnosis & $23(74.2)$ \\
\hline At lenalidomide initiation & $31(100)$ \\
\hline \multicolumn{2}{|l|}{ Karyotype } \\
\hline Isolated del(5q) only & $28(90.3)$ \\
\hline+1 additional abnormality & $3(9.7)$ \\
\hline \multicolumn{2}{|l|}{ WHO classification } \\
\hline MDS with isolate del(5q) & $27(87.1)$ \\
\hline MDS, multilineage dysplasia & $2(6.5)$ \\
\hline MDS, excess blast- 1 & $1(3.2)$ \\
\hline MDS, unclassifiable & $1(3.2)$ \\
\hline \multicolumn{2}{|c|}{ International Prognosis Scoring System (IPSS) } \\
\hline Low & $16(51.6)$ \\
\hline Intermediate-1 & $15(48.4)$ \\
\hline Intermediate-2 or high & $0(0 \%)$ \\
\hline \multicolumn{2}{|l|}{ IPSS-revised (IPSS-R) } \\
\hline Very low & $2(6.5)$ \\
\hline Low & $16(51.6)$ \\
\hline Intermediate & $15(41.9)$ \\
\hline High/very high & $0(0)$ \\
\hline
\end{tabular}

Abbreviaion: MDS, myelodysplastic syndrome. rical variables are summarized in the tables. Fisher's exact test or Pearson's chi-squared test was performed to determine the non-random associations between the two categorical variables. The data for the continuous variables are expressed as median, range (minimum-maximal value), and mean $\pm S E M$. The values were two-sided, and statistical significance was set at $P<0.05$.

\section{RESULTS}

\section{Patient characteristics}

A total of 31 patients from 21 institutions were included in the study. Of them, 30 patients were native Koreans and 1 patient was a Chinese resident in Korea. There was a female predominance, and the median age of the patients at the time of the first exposure to lenalidomide was 67 years (range, 46-83 yr). The time interval from MDS diagnosis to the first exposure to lenalidomide varied as some patients had to wait for Korea MFDS approval and reimbursement for using lenalidomide to treat their disease. In addition, 12 of 31 patients $(38.7 \%)$ were previously treated with erythropoiesis-stimulating agents (ESAs), and only 4 of them achieved RBC-TI (Supplementary Table 1), reflecting the poor response to ESAs for del(5q) MDS [17]. The patient characteristics are summarized in Table 1.

All 31 patients showed RBC transfusion dependence at the time of lenalidomide initiation. The baseline hemoglobin $(\mathrm{Hb})$ level had a median value of $7.0 \mathrm{~g} / \mathrm{dL}$ (range, $4.5-10.0$ ) and a mean value of $7.2 \pm 0.2 \mathrm{~g} / \mathrm{dL}$. For RBC transfusion 8 weeks before lenalidomide initiation, the median number of units was 6 (range, 2-11), and the mean number of units was $5.3 \pm 0.4$ units.

\section{Efficacy of lenalidomide}

The response of the three patients could not be evaluated. All of them discontinued lenalidomide after less than 28 days for the following reasons: toxicity (1 patient; in 18

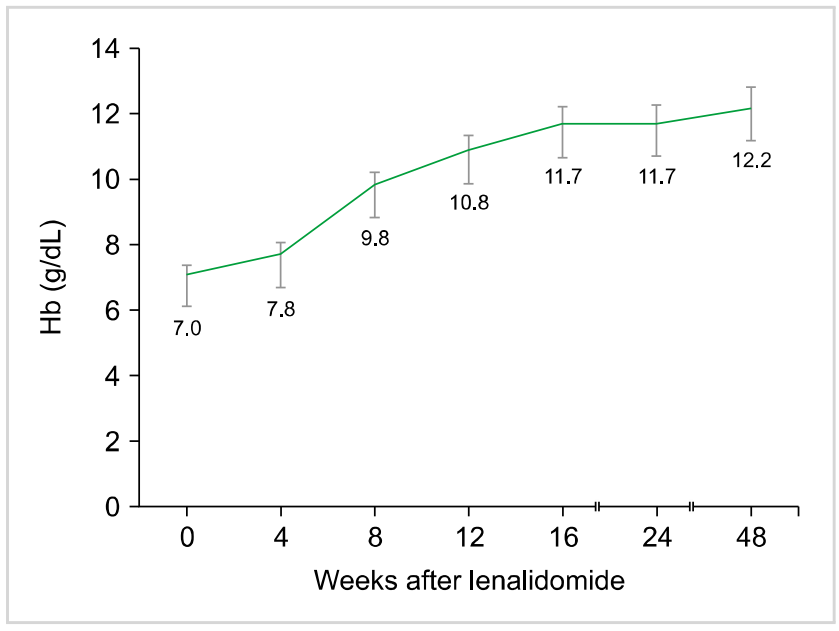

Fig. 1. Hemoglobin level changes in responders after lenalidomide treatment (mean \pm SEM). 
days), rapid AML transformation (1 patient; in 20 days), and at will (1 patient, in 22 days). Of the remaining 28 patients, 19 (67.9\%) achieved RBC-TI. The other 9 patients were non-responsive to lenalidomide, including one patient with a minor HI-E response ( $\geq 50 \%$ reduction in RBC transfusion but without independence). A follow-up bone marrow examination was performed for only 2 patients, both of whom achieved RBC-TI and showed partially decreased del(5q) cytogenetic abnormality with morphologic complete response.

The duration of lenalidomide use was significantly longer among responders (mean $24.7 \pm 2.5 \mathrm{mo}$ ) than among non-responders (mean $9.1 \pm 2.6 \mathrm{mo} ; P<0.001$ ). At the time of data cutoff, all responders were still using lenalidomide. Among the responders, the mean $\mathrm{Hb}$ level began to increase during the 4 th week after treatment initiation, showed a sharp increase from the 4 th to 8 th week, and maintained a gradually increasing trend thereafter (Fig. 1).

Female sex and the development of thrombocytopenia during treatment were associated with achieving RBC-TI. Younger age, lower pre-treatment RBC transfusion burden, and higher ANC and platelet count showed a tendency toward achieving RBC-TI; however, the results were not statistically significant (Table 2).

\section{Tolerability of lenalidomide}

All 31 patients were evaluated for toxicity (Table 3). Overall, $22(71.0 \%)$ and $8(25.8 \%)$ patients had grade 3 or 4 neutropenia and thrombocytopenia, respectively. The most common non-hematologic toxicities were pruritus, fatigue, and rashes. Grade 3 or 4 non-hematologic toxicities were limited to rash (4 patients; $12.9 \%$ ) and pruritus (2 patients; $6.5 \%)$.

A dose reduction in lenalidomide was required for 15 of 19 responders (78.9\%) and 3 of 9 non-responders (33.3\%).
A dose interruption in lenalidomide was required for 7 of 19 responders (36.8\%). A total of 12 patients eventually discontinued lenalidomide for the following reasons: no response (6 patients), intolerable toxicity under a non-responsive state (4 patients), rapid AML progression (1 patient), and at will (1 patient).

The most common final stable dosing schedule of lenalidomide for the 19 responders was $5 \mathrm{mg}$ per os once every other day ( $\mathrm{N}=6,31.6 \%$; Table 4$)$. There was no significant association between the final dose intensity of lenalidomide and the body mass index $(\mathrm{r}=0.133, P=0.588)$ or bodyweight (r=0.325, $P=1.000)$ of the patients.

\section{DISCUSSION}

In this study, we demonstrated acceptable lenalidomide efficacy and tolerability in $\operatorname{del}(5 \mathrm{q})$ MDS patients of East Asian ethnicity. The RBC-TI rate of $67.9 \%$ is comparable to that reported in western studies, which is encouraging considering that it is based on real-world data.

Our main objective was to determine whether Asian patients, who have lower body mass indexes than westerners [18], have a higher incidence and severity of toxicity with the current standard dose of lenalidomide. Even among Westerners, frequent dose reduction and interruption have been reported for lenalidomide [11, 12, 19]. The toxicities were generally mild and manageable, with adequate supportive care. The incidence of hematologic toxicities was similar to that reported in previous studies. Febrile neutropenia was observed in $9.7 \%$ of patients, and all patients recovered without sequelae, suggesting that the risk of infectious complication was not high. Nevertheless, care should be taken in older adults or comorbid patients. The profiles of non-hematologic toxicities were in agreement with the western data,

Table 2. Evaluation of potential predictors for RBC-TI.

\begin{tabular}{|c|c|c|c|}
\hline & Response no & Response yes & $P$ \\
\hline Male & 5 & 2 & 0.020 \\
\hline Female & 4 & 17 & \\
\hline Age $<$ median & 7 & 8 & 0.086 \\
\hline Age $\geq$ median & 2 & 11 & \\
\hline MDS diagnosis to lenalidomide initiation $<2 \mathrm{yr}$ & 4 & 11 & 0.410 \\
\hline MDS diagnosis to lenalidomide initiation $\geq 2 \mathrm{yr}$ & 5 & 8 & \\
\hline IPSS low & 5 & 10 & 0.604 \\
\hline IPSS intermediate-1 & 4 & 9 & \\
\hline Pre-treatment red blood cell transfusion $\leq 4 \mathrm{U} / 8 \mathrm{wk}$ & 3 & 11 & 0.210 \\
\hline Pre-treatment red blood cell transfusion $>4 \mathrm{U} / 8 \mathrm{wk}$ & 6 & 8 & \\
\hline Pre-treatment neutrophil $\geq 1,000 / \mu \mathrm{L}$ & 5 & 16 & 0.123 \\
\hline Pre-treatment neutrophil $<1,000 / \mu \mathrm{L}$ & 4 & 3 & \\
\hline Pre-treatment platelet $\geq 150 \mathrm{~K} / \mu \mathrm{L}$ & 5 & 17 & 0.064 \\
\hline Pre-treatment platelet $<150 \mathrm{~K} / \mu \mathrm{L}$ & 4 & 2 & \\
\hline Development of thrombocytopenia during treatment & 1 & 7 & 0.038 \\
\hline No development of thrombocytopenia during treatment & 8 & 12 & \\
\hline
\end{tabular}

Abbreviation: IPSS, International prognostic scoring system. 
Table 3. Hematologic and non-hematologic toxicities (worst during treatment).

\begin{tabular}{|c|c|c|c|c|c|c|c|}
\hline Toxicity & None & Grade 1 & Grade 2 & Grade 3 & Grade 4 & Any (\%) & Grade 3/4 (\%) \\
\hline \multicolumn{8}{|l|}{ Hematologic } \\
\hline Neutropenia & 7 & 1 & 1 & 13 & 9 & $24(77.4)$ & $22(71.0)$ \\
\hline Lymphopenia & 20 & 5 & 3 & 3 & 0 & $8(25.8)$ & $3(9.7)$ \\
\hline Thrombocytopenia & 11 & 9 & 3 & 3 & 5 & $20(64.5)$ & $8(25.8)$ \\
\hline Febrile neutropenia & 28 & $-{ }_{-}^{\text {a) }}$ & -a) & 3 & 0 & $3(9.7)$ & $3(9.7)$ \\
\hline \multicolumn{8}{|l|}{ Non-hematologic } \\
\hline Pruritus & 16 & 8 & 5 & 2 & 0 & $15(48.4)$ & $2(6.5)$ \\
\hline Fatigue & 17 & 12 & 2 & 0 & 0 & $14(45.2)$ & 0 \\
\hline Rash & 22 & 5 & 0 & 4 & 0 & $9(29.0)$ & $4(12.9)$ \\
\hline Diarrhea & 26 & 3 & 2 & 0 & 0 & $5(16.2)$ & 0 \\
\hline Dyspepsia & 28 & 1 & 2 & 0 & 0 & $3(9.7)$ & 0 \\
\hline Constipation & 29 & 2 & 0 & 0 & 0 & $2(6.5)$ & 0 \\
\hline Headache & 30 & 0 & 1 & 0 & 0 & $1(3.2)$ & 0 \\
\hline Insomnia & 30 & 0 & 1 & 0 & 0 & $1(3.2)$ & 0 \\
\hline Deep vein thrombosis & 30 & 0 & 1 & 0 & 0 & $1(3.2)$ & 0 \\
\hline Dyspnea & 30 & 1 & 0 & 0 & 0 & $1(3.2)$ & 0 \\
\hline Tongue discoloration & 30 & 1 & 0 & 0 & 0 & $1(3.2)$ & 0 \\
\hline Loss of appetite & 30 & 1 & 0 & 0 & 0 & $1(3.2)$ & 0 \\
\hline Increased creatinine & 30 & 0 & 1 & 0 & 0 & $1(3.2)$ & 0 \\
\hline
\end{tabular}

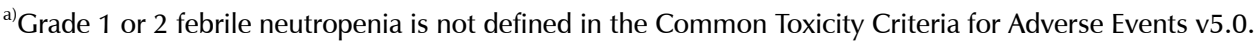

Table 4. Final stable dose of lenalidomide for responders $(\mathrm{N}=19)$.

\begin{tabular}{lcc}
\hline \multicolumn{1}{|c}{ Lenalidomide dosing } & $\begin{array}{c}\text { Patient } \\
\text { No. }\end{array}$ & $\begin{array}{c}\text { Dose intensity } \\
(\mathrm{mg} / \text { day })\end{array}$ \\
\hline $10 \mathrm{mg}$ once daily & 1 & 10 \\
$10 \mathrm{mg}$ once daily for $3 \mathrm{wk}, 1$-wk rest & 3 & 7.5 \\
$10 \mathrm{mg}$ once every other day & 1 & 5 \\
$5 \mathrm{mg}$ once daily & 4 & 5 \\
$5 \mathrm{mg}$ once daily for $3 \mathrm{wk}, 1$-wk rest & 4 & 3.75 \\
5 mg once every other day & 6 & 2.5 \\
\hline
\end{tabular}

except for the lower incidence and severity of venous thromboembolism (VTE). In a randomized phase 3 trial, the most common non-hematologic grade 3 or 4 adverse events were venous thrombosis (4 of 69 patients in the $10 \mathrm{mg}$ group) and one death due to pulmonary embolism [12]. In addition, the French GFM group reported that 8 of 95 patients $(9.5 \%)$ developed VTE after a median of 16 weeks (range, 8-90 wk) of treatment [19]. In contrast, our study included only one patient (3.2\%) with VTE, who was a 60 -year-old woman with deep vein thrombosis of the left upper proximal extremities after 12 weeks of treatment. In general, patients with del(5q) MDS have thrombophilic risk factors, including older age, female predominance, higher baseline platelet count, increased hemoglobin level after lenalidomide use, and previous exposure to ESAs. Although a careful interpretation of the results should be made due to the limited sample size, the difference may be attributed to the lower incidence of VTE in Asian populations [20].

In the MDS-003 trial [11], there were no differences be- tween the efficacy and safety profiles of the two dosing schedules of lenalidomide (10 mg/day on days 1 to 28 vs. $10 \mathrm{mg}$ /day on days 1 to 21 followed by 7 days of rest). In the phase 3 MDS-004 trial [12], a smaller dose (5 mg/day on days 1 to 28 ) was compared to $10 \mathrm{mg}$ /day for 21 days. Although there was no significant difference between 10 $\mathrm{mg} /$ day for 21 days and $5 \mathrm{mg} /$ day for 28 days, the $10 \mathrm{mg} /$ day for 21 days dose was recommended because it had some advantages. However, after this starting dose, subsequent dose reductions were common, ranging from $48 \%$ to $84 \%$ $[11,12,19]$. In our study, 18 of 28 evaluable patients (64.3\%) required dose reduction, and the most common stable dose was $5 \mathrm{mg}$ once every other day. Interestingly, the rate of dose adjustment was higher among responders than among non-responders $(78.9 \%$ vs. $33.3 \%, P=0.03)$, suggesting that dose reduction did not result in a suboptimal response. A dose reduction in lenalidomide due to toxicity was predictable and common among responders; thus, there was no need to hesitate when it was necessary during treatment.

We found no association between the body mass indexes or bodyweights of patients and the stable dose of lenalidomide. Moreover, the overall incidence and severity of adverse events in our study were not significantly greater than those reported by western studies. This suggests that patients with a lean body mass are not more vulnerable to lenalidomide-related toxicities than those without a lean body mass. Based on the results, an identical initial dosing schedule should be applied to Asian populations with the readiness to provide timely and appropriate dose adjustment and supportive care in response to the development of adverse events.

A drop in the platelet count during treatment, particularly 
after lenalidomide use, is known to be associated with the erythroid response, indicating the successful reduction of MDS clones due to the synthetic lethal activity of lenalidomide. This effect was also observed in the present study. Female sex was associated with the achievement of RBC-TI. However, the result was not conclusive because the patients, especially the male patients, were few.

Although there were no deaths and one patient had rapid AML progression, a robust evaluation of the long-term disease course and survival was not feasible due to the short follow-up period. This may be considered a limitation of the current study. Nevertheless, we successfully demonstrated that RBC-TI could be effectively achieved with lenalidomide monotherapy in Asian del(5q) MDS patients.

In conclusion, lenalidomide efficacy and tolerability were similar in Asian del(5q) MDS patients and western patients. A dose reduction in lenalidomide during treatment was common and was not associated with inferior outcomes.

\section{Authors' Disclosures of Potential Conflicts of Interest}

No potential conflicts of interest relevant to this article were reported.

\section{REFERENCES}

1. Cazzola M. Myelodysplastic syndromes. N Engl J Med 2020;3833: 1358-74.

2. Greenberg P, Cox C, LeBeau MM, et al. International scoring system for evaluating prognosis in myelodysplastic syndromes. Blood 1997;89:2079-88.

3. Greenberg PL, Tuechler H, Schanz J, et al. Revised international prognostic scoring system for myelodysplastic syndromes. Blood 2012;120:2454-65.

4. Fenaux $P$, Adès L. How we treat lower-risk myelodysplastic syndromes. Blood 2013;121:4280-6.

5. Choi EJ, Lee JH, Lee JH, et al. Treatment and clinical outcomes of patients relapsing after allogeneic hematopoietic cell transplantation for myelodysplastic syndrome. Blood Res 2018;53:288-93.

6. Haase D. Cytogenetic features in myelodysplastic syndromes. Ann Hematol 2008;87:515-26.

7. Adema V, Bejar R. What lies beyond del $(5 q)$ in myelodysplastic syndrome? Haematologica 2013;98:1819-21.
8. Arber DA, Orazi A, Hasserjian R, et al. The 2016 revision to the World Health Organization classification of myeloid neoplasms and acute leukemia. Blood 2016;127:2391-405.

9. Boultwood J, Pellagatti A, McKenzie AN, Wainscoat JS. Advances in the 5q- syndrome. Blood 2010;116:5803-11.

10. Sperling AS, Gibson CJ, Ebert BL. The genetics of myelodysplastic syndrome: from clonal haematopoiesis to secondary leukaemia. Nat Rev Cancer 2017;17:5-19.

11. List A, Dewald G, Bennett J, et al. Lenalidomide in the myelodysplastic syndrome with chromosome $5 q$ deletion. $N$ Engl J Med 2006;355:1456-65.

12. Fenaux $\mathrm{P}$, Giagounidis A, Selleslag D, et al. A randomized phase 3 study of lenalidomide versus placebo in RBC transfusiondependent patients with Low-/Intermediate-1-risk myelodysplastic syndromes with del5q. Blood 2011;118:3765-76.

13. Kuendgen A, Lauseker M, List AF, et al. Lenalidomide does not increase AML progression risk in RBC transfusion-dependent patients with Low- or Intermediate-1-risk MDS with del(5q): a comparative analysis. Leukemia 2013;27:1072-9.

14. Lee JH, Lee JH, Shin YR, et al. Application of different prognostic scoring systems and comparison of the FAB and WHO classifications in Korean patients with myelodysplastic syndrome. Leukemia 2003;17:305-13.

15. Miyazaki Y, Tuechler H, Sanz G, et al. Differing clinical features between Japanese and Caucasian patients with myelodysplastic syndromes: analysis from the International Working Group for Prognosis of MDS. Leuk Res 2018;73:51-7.

16. Harada $\mathrm{H}$, Watanabe $\mathrm{M}$, Suzuki $\mathrm{K}$, et al. Lenalidomide is active in Japanese patients with symptomatic anemia in low- or intermediate- 1 risk myelodysplastic syndromes with a deletion $5 \mathrm{q}$ abnormality. Int J Hematol 2009;90:353-60.

17. Kelaidi C, Park S, Brechignac S, et al. Treatment of myelodysplastic syndromes with $5 \mathrm{q}$ deletion before the lenalidomide era; the GFM experience with EPO and thalidomide. Leuk Res 2008;32:1049-53.

18. Pan WH, Flegal KM, Chang HY, Yeh WT, Yeh CJ, Lee WC. Body mass index and obesity-related metabolic disorders in Taiwanese and US whites and blacks: implications for definitions of overweight and obesity for Asians. Am J Clin Nutr 2004;79:31-9.

19. Le Bras F, Sebert M, Kelaidi C, et al. Treatment by lenalidomide in lower risk myelodysplastic syndrome with $5 q$ deletion--the GFM experience. Leuk Res 2011;35:1444-8.

20. Hong J, Lee JH, Yhim HY, et al. Incidence of venous thromboembolism in Korea from 2009 to 2013. PLoS One 2018;13: e0191897. 